Background Newborns frequently need to be transferred to level-IIIcenters shortly after birth. Especially in very low birth weight infants, these postnatal transports are correlated with increased risk for intraventricular hemorrhage. Despite specifically designed incubators, infants are exposed to a considerable amount of mechanical vibrations $(\mathrm{MV})$ that are presumed to be one of the major risk factors.

Aims The objective was to investigate the extent of MV and the frequency spectrum occurring during neonatal transport.

Methods Two types of incubators (ITI-5400 (INC1), Air-ShieldsIsolette-TI-500 (INC2), both DRÄGER, Lübeck, Germany) were tested during simulated neonatal transport by ambulance vehicle on various road types. MV were recorded by highly-sensitive accelerometers (LIS331DL, STMicroelectronics, Geneva, Switzerland) and analyzed by using Fast-Fourier-Transform and Continuous-Wavelet-Transform.

Results MV occurring in INC1 during transport had peak accelerations up to $0.91 \mathrm{~g}\left(8.93 \mathrm{~m} / \mathrm{s}^{2}\right)$ and a dominant frequency range of 10-14 Hertz. Measurements with INC2 showed significantly higher peak accelerations with values up to $1.60 \mathrm{~g}\left(15.70 \mathrm{~m} / \mathrm{s}^{2}\right)$ and further a distinct peak in the frequency spectrum at approximately 15 Hertz. Total detected MV within the investigated frequency band of 1-50 Hertz were up to 8 times higher in INC2 compared to INC1. Conclusions We were able to demonstrate that during neonatal transport newborns might be exposed to almost twofold gravitational acceleration, whereby accelerations and vibration frequencies differed distinctly between incubators. On the basis of these data implementation of vibration analysis in approval procedures of transport incubators has to be considered.

\section{NEONATAL OUTCOMES OF VERY PREMATURE INFANTS BORN AFTER IN VITRO FERTILIZATION}

doi:10.1136/archdischild-2012-302724.1758

T Teresa del Moral, S Dominguez, K Kooning, S Vanburskit. Pediatrics, University of Miami Miller School of Medicine, Miami, FL, USA

Advances in assisted reproductive technology such as in vitro fertilization (IVF) is known to be associated with a high rate of multiple pregnancy and prematurity. However reliable data on neonatal outcomes of infants born preterm after IVF are lacking.

A cohort study was conducted to compare neonatal outcomes of 90 very premature infants born after IVF at University of Miami/ Jackson Memorial Hospital between 2005 and 2011, with a control group born after natural conception, matched by gestational age. The IRB approved the study.

Mothers of infants born after IVF were older (34 vs 28 yrs $\mathrm{p}<0.001)$ and had lower parity (0 vs $1 \mathrm{p}<0.001)$. Gestational age and birth weight were not different between groups. More IVF infants were females ( $56 \%$ vs $40 \% \mathrm{p}<0.01)$ and were born by C-section ( $94 \%$ vs $88 \% \mathrm{p}<0.03)$. The only difference between groups was a higher 1 minute Apgar score in IVF infants (6.2 vs $5.1 \mathrm{p}<0.002)$. No differences in 5 and 10 minutes Apgar's score, need for surfactant, duration of mechanical ventilation and need for $\mathrm{O}_{2}$ were found. There were no differences in the incidence of major morbidities, IVH, BPD, NEC, sepsis, and PDA ligation. Mortality and LOS were similar in both groups.

These results demonstrate that when compared with infants of similar gestational age outcomes of IVF premature infants are not different from naturally conceived infants. The reported higher risk for poor outcomes in IVF infants is most likely related with the higher risk of multiple gestation and prematurity.

\section{REDUCTION IN CENTRAL LINE ASSOCIATED BLOODSTREAM INFECTIONS BY INTRODUCING A QUALITY IMPROVEMENT PATHWAY 'CLEAN LINE'}

doi:10.1136/archdischild-2012-302724.1759
OJ Kleinlugtenbeld, HLM van Straaten, MI van den Bos, MAC Hemels, EJ d'Haens. Neonatology, Isala Clinics, Zwolle, The Netherlands

Background Central lines (umbilical arterial/venous catheter, central venous catheter) are commonly used for NICU patients.

The most common complication is the Central Line Associated nosocomial Bloodstream Infection (CLABSI).

Reducing CLABSIs improves short and long term outcome for premature newborn.

Implementing bundles of care may reduce CLABSIs.

Aim Does implementation of "bundles of care" reduce CLABSIs/1000 catheter days?

Methods In 2010 a task group 'Clean Line' (neonatologist, Nurse Practitioner, hygienist, NICU nurse and ward manager) defined five bundles of care:
1. optimal insertionconditions,
2. handhygiene,
3. daily line care,
4. daily review of line necessity and
5. daily inspection of insertion site.

For each carebundle the procedures were evaluated on an evidence-based manner and changed where needed. Video demonstrating best practice, multidisciplinary education and short checklists for monitoring compliance were used for implementation.

In 2011 the pathway started with the first two bundles of care. Data of CLABSIs and catheter days were compared with a historical cohort (2007). CLABSI is defined as clinical sepsis $>72$ hours after birth with a positive blood culture without other focus.

Results

Abstract 1759 Table 1

\begin{tabular}{lll}
\hline Number & $\mathbf{2 0 0 7}$ & $\mathbf{2 0 1 1}$ \\
\hline Patients (catheters) & $75(124)$ & $206(345)$ \\
Catheterdays (no.) & 795 & 1727 \\
CLABSI (no) & 16 & 11 \\
CLABSI/1000 days & $20,1^{1}$ & $6.3^{1}$ \\
\hline
\end{tabular}

'Difference 13.8 (Cl 3.2-24.3).

Conclusion A NICU quality improvement pathway with implementation of bundles of care can reduce the number of catheterrelated infections/1000 catheter days.

\section{NEONATAL RE-ADMISSIONS WITH FEEDING DIFFICULTIES IN A LARGE DISTRICT -GENERAL HOSPITAL IN UK: MORE SUPPORT NEEDED FOR BREASTFEEDING MOTHERS?}

doi:10.1136/archdischild-2012-302724.1760

K Annam, H Alabede, S Rajendran, A Shastri. Colchester General Hospital, Colchester Hospital University NHS Trust, Colchester, UK

Background and Aims Readmission of neonates due to weight loss and feeding difficulties to hospital following discharge continues to be a concern. This audit was done to study the incidence, clinical characteristics and laboratory markers of neonates admitted with feeding difficulties and weight loss

Methods Clinical notes and discharge summaries of babies $\leq 28$ days old admitted to hospital over a period of ten months from June 2011 to March 2012 were reviewed. Information was recorded regarding age at admission, weight loss, final diagnosis and feeding method.

Results A total of 114 neonates were re-admitted to hospital during this period. Of these 34 (30\%) were due to feeding difficulties \pm jaundice. 29/34 of these were due to weight loss related to breastfeeding. Other 5 neonates were bottle fed and needed treatment for jaundice \pm supervision of feeding in hospital. Among the 29 breast fed babies, 19 (65\%) had significant weight loss of $>10 \%$, 9 had 
$10-14 \%$ and 10 had $\geq 15 \%$. The median age at presentation was 5 days (2-12 days), median sodium level with $>15 \%$ weight loss babies was 152 (143-162).

Conclusions A significant number of neonatal re-admissions were due to abnormal weight loss of $\geq 10 \%$ and majority were due to failure to establish breastfeeding. An earlier audit in 2009 had identified need for more breast feeding support. Although systems to support breast feeding mothers in community are in place, more support needs to be established including regular assessment of weight to avoid hospital re-admissions.

\section{PITFALLS OF THE NEONATAL SCREENING PROGRAM FOR CONGENITAL ADRENAL HYPERPLASIA (CAH)}

doi:10.1136/archdischild-2012-302724.1761

HA Abu Hamdan, F Sabbagh, MM Abou Al-Seoud. Neonatal Division - Paediatric Department, King Fahad Armed Forces Hospital, Jeddah, Saudi Arabia

Background Prompt diagnosis and treatment of CAH is essential to prevent mortality and morbidity. The incidence of $\mathrm{CAH}$ ranges from 1 in 21270 (New Zealand) to 1 in 5000 (Saudi Arabia).

Our Aim:

Identify the incidence of $\mathrm{CAH}$ in our patient population.

Appraise the $\mathrm{CAH}$ screening program.

Method Retrospective analysis of filter paper blood samples from infants aged 36 hours or more collected from November 2007 - Sept 2011. The 17-hydroxyprogesterone (17-OHP) tests were part of metabolic screening program at KFAFH. Abnormal values were re-called.

Results 22381 of 22428 (99.7\%) births were screened for CAH. The turnaround time was 10 days. 124 infants [males 89 (72\%) females $31(28 \%)$ ] had abnormal levels and were re-called (recall rate $0.55 \%$ ). 76 infants had repeated serum 17-OHP concentration (response rate $61 \%$ ). 7 infants had abnormal elevated levels, 4 had ambiguouse genitalia(karyotype female), 3 male infants had symptoms of salt wasting at age of 9.10 and 37 days, turnaround time for the screening results was 11.9 and 16 days respectively. Delayed identification was due to failed contact and response in case 2 and 3 respectively. Recall failure was in 48/124 (39\%) reasons were wrong contact numbers in 36 cases $(75 \%)$ and no show in 10 cases $(21 \%)$. Failure to repeat in 2 preterm infants died secondary to prematurity.

Conclusion The incidence of CAH is 1 in 3333 in our patient population. Barriers for timely intervention were due to prolonged turnaround time, lack of family education. Strategies to improve the process should be implemented.

\section{CURRENT RESUSCITATION PRACTICES AMONG PEDIATRICIANS IN GUJARAT, INDIA - EFFECT OF 2010 RESUSCITATION GUIDELINES}

doi:10.1136/archdischild-2012-302724.1762

'AS Nimbalkar, 'SC Bansal, 'DV Patel, 'AR Sethi, 1,2SM Nimbalkar. 'Department of Pediatrics, Pramukhswami Medical College; 'Central Research Services, Charutar Arogya Mandal, Anand, India

Background and Aims Neonatal Resuscitation Guidelines are updated by international societies every five years. India does not have mandatory certification and hence many pediatricians may not change their practices which may be in variance with current guidelines. We aimed to see the acceptance and awareness of several recently incorporated practices in resuscitation.

Methods Questionnaire based cross-sectional study administered by a web based form the link of which was sent by email and/or social networking sites to 600 pediatricians in state of Gujarat. A total of 25 questions were asked requiring about 10 minutes of the respondents time.
Results 75 pediatricians responded with 62 usable questionnaires. About $83 \%$ participants worked in a Level $2 / 3$ NICU. $64 \%$ had resuscitated more than 20 neonates and $78 \%$ had attended more than 100 deliveries. Hence respondents were attending deliveries and required skills in resuscitation. $81.3 \%$ used bag and mask with only $33.3 \%$ using room air for resuscitation. $58.5 \%$ were using correct rate of ventilation. $76 \%$ had saturation monitors but only $17.2 \%$ had oxygen blenders. $31.7 \%$ approved of self inflating bag for free flow oxygen. $50 \%$ judged adequacy of ventilation by chest rise against $41.9 \%$ who used rising heart rate. Only about $50 \%$ pediatricians had undergone a training course in past 3 years. $41.7 \%$ believed in immediate cord cutting for normal deliveries. Only $32.3 \%$ responded correctly on the duration of resuscitation for asystole.

Conclusions The knowledge of pediatricians regarding resuscitation of neonates is poor. Only few pediatricians update themselves by attending courses.

\section{NEWBORN HEARING SCREENING: THE EXPERIENCE OF THE UNIVERSITY HOSPITAL OF MODENA}

doi:10.1136/archdischild-2012-302724.1763

${ }^{1} \mathrm{~S}$ Palma, ${ }^{2} \mathrm{MF}$ Roversi, ${ }^{3} \mathrm{FM}$ Artioli, ${ }^{3} \mathrm{G}$ Denti, ${ }^{2} \mathrm{~F}$ Ferrari, ${ }^{3} \mathrm{E}$ Genovese. ${ }^{1}$ Primary Care Department, Azienda ASL; ${ }^{2}$ Department of Neonatology, Neonatal Intensive Care Unit, Modena University; ${ }^{3}$ Ent Department, Audiology, Modena University, Modena, Italy

Background and Aims Newborn hearing screening programs allow to identify infants with bilateral permanent congenital hearing impairment and to facilitate early intervention to minimize the consequences on language development. In our country the concept of implementing universal newborn hearing screening protocols is still a topic of debate. Our aim was to analyze the first data collected in one year of experience since the adoption of the universal newborn hearing screening in Modena University Hospital.

Methods Data were collected during the period from $8^{\text {th }}$ April 2011 to $31^{\text {st }}$ March 2012. The screening was carried out by means of Transient Stimulus Evoked Otoacoustic Emissions, using, for well babies, a two-stage protocol: first screening stage on the second day after birth, followed by re-screening before discharge if a pass response was not obtained from both ears and a second stage follow up screening within 3 weeks later in case of failure. In case of a persistent failure response an audiologic evaluation was performed.

Results During the period of the study 3512 babies underwent newborn hearing screening, of these 203 presented increased risk factors. In well babies group, one child will undergo surgery for a cochlear implant, 3 children are receiving a prosthetic-rehabilitative treatment. The prevalence of permanent bilateral hearing loss resulted 1.2:1000.

Conclusion It is important to create an active collaboration between audiologists/ENT specialists and paediatricians to reach the objective of identifying infants with hearing loss as early as possible in order to implement early interventions.

\section{INVESTIGATION OF SOME ASPECTS OF PERCEIVED SOCIAL SUPPORT IN MOTHERS OF HOSPITALIZED CHILDREN IN NEONATAL INTENSIVE CARE UNIT}

doi:10.1136/archdischild-2012-302724.1764

'S Kara, ${ }^{2} \mathrm{~S}$ Tan, ${ }^{2} \mathrm{~S}$ Aldemir, ${ }^{3} \mathrm{AE}$ Y Ylmaz, ${ }^{3} \mathrm{MM}$ Tatll, ${ }^{4} \mathrm{U}$ Dilmen. 'Department of Neonatology, Ankara Training and Research Hospital; '2Department of Psychiatry; ${ }^{3}$ Department of Neonatology, Fatih University Medical Faculty; ${ }^{4}$ Department of Neonatology, Zekai Tahir Burak Maternity and Teaching Hospital, Ankara, Turkey

Purpose To identify the degree of perceived social support in mothers of hospitalized children in neonatal intensive care unit, and to assess in psychosocial aspects the relationship of the perceived social support with such variables as depression and anxiety level. 